\title{
Editorial: Adaptive Communication in Wireless Networks
}

\author{
Sudip Misra · Issa Traore · Wei Song
}

Published online: 28 April 2010

(C) Springer Science+Business Media, LLC. 2010

Wireless communication technologies are undergoing rapid advancements. The last few years have experienced a steep growth in research on wireless networks having attractive claims. Researchers are currently envisioning different attractive properties of wireless systems such as the ability to self-organize, self-configure, self-heal, self-manage and self-maintain. These systems are increasingly becoming dynamic and they are expected to perform many tasks autonomously by adapting to the dynamics of the networks. With the wide range of applications that need to be supported in these systems, there are increasing expectations about what the current and the future generation networks can do. Many of these requirements are pivoted in the ability of the networks to adapt to the network dynamism. Adaptation to changing environments, in turn, often leads to increased performance of the networks. In this Special Issue, we have selected a few high quality research papers relating to different aspects of adaptive communication in wireless networks. We provide a cursory level summary of the papers.

Guo et al. have proposed an energy efficient multi-source temporal data aggregation model called MSTDA in WSNs. This MSTDA model is deployed at both the base station (BS) and the node. In this MSTDA model they included feature selection and data prediction methods. Experimental results shown in the proposed method provide good performance.

Deng et al. have proposed a collision alleviation scheme for the purpose of efficient high priority traffic transmission which usually used to schedule emergency messages in VANETs. The simulation results show that there is significant improvement in performance for high priority traffic without much degraded performance of low priority traffic.

S. Misra $(\varangle)$

School of Information Technology, IIT Kharagpur, Kharagpur, India

e-mail: smisra.editor@gmail.com

I. Traore

Department of Electrical and Computer Engineering, University of Victoria, Victoria, Canada e-mail: itraore@ece.uvic.ca

W. Song

Faculty of Computer Science, University of New Brunswick, Bathurst, Canada

e-mail:wsong@unb.ca 
Vaidya et al. have proposed a comprehensive and resilient security framework for wireless ad hoc network capable of providing end to end security. They have proposed an approach using a multi-signatures scheme along with a self-certified public keying technique. The experimental results show that it is more secure and effective than the existing schemes.

Li et al. have proposed an optimization based adaptive routing algorithm which can be used for seeking a desired path for traffic flows. They have formulated a convex multi-commodity network flow problem. The experimental results show that it is more robust and adaptable for a variety of network situations.

Fei et al. have proposed a QOS aware two level uplink DBA algorithm for IEEE 802.16jbased vehicular networks. The proposed DBA algorithm consists of two levels DBA algorithm. The simulation results show that the proposed DBA algorithm operates efficiently in terms of the average queuing delay, call blocking probability and throughput.

Tsai et al. proposed an adaptive packet and block length forward error correction (FEC) for video streaming over wireless networks. They have shown that the proposed scheme can obtain better recovery performance compared to the conventional FEC mechanisms, which use a whole redundant packet to recover the error source packet, when the packet error occurs with only a few bit errors inside.

Sarker et al. have proposed a new access protocol with Multi-packet Reception (MPR) capability for infrastructure less wireless autonomic networks. In an infrastructure less wireless autonomic network, mobile nodes may communicate with each other directly without a base station, using the proposed random access protocol. The results of this study may be used for a system design of an infrastructure less contention based multiple access schemes with MPR capability.

Liu et al. have studied the symbol error rate (SER) performance of adaptive relay selection schemes in general dual hop multiple relay networks. They have derived tight and closed form results over the whole SNR regime for ARS, AF-RS, PDF-RS, ADF-RS, and CDF-RS schemes.

Shahriar et al. have presented analytical model based analysis and evaluation tool for PD-based route optimization schemes. Results show that the performance of a scheme depends on the characteristics of the mobile network, and there is no single scheme which suits all mobility scenarios.

Song has analyzed the impact of channel fading, direct collisions, and staggered collisions on MAC-layer packetization in 802.11 WLANs. A large packet size is preferred to minimize protocol header overhead. A significant performance gain is observed in terms of effective throughput and video frame transfer delay.

OFDM technology is expected to play important role in future wireless communication systems. The performance analysis of OFDM-based cooperative networks over Nakagami- $m$ fading channels is a necessary step in the design of future OFDM-based cooperative networks. In this special issue, Yang and colleagues have investigated (for the first time) the performance of OFDM-based cooperative networks over Nakagami- $m$ fading channels, by deriving the closed-form outage probability of OFDM-based selective DF cooperative networks over independent but not necessarily identically distributed (i.n.i.d.) Nakagami- $m$ fading channels, with integer values of parameter $m$.

Pillutla and Krishnamurthy have tackled the problem of decentralized rate selection in IEEE 802.11 wireless local area networks (WLANs) using game theory. Several interesting results have been established by formulating the rate selection problem as a non-cooperative game where individual users (players) of a WLAN can pick their actions from a finite set of physical layer modulation rates. 
Qiu and colleagues address the poor performance faced by some emerging applications with high bandwidth requirement and delay and loss constraints, such as video streaming, in Wireless Mesh Networks (WMNs). They developed a new approach consisting of a network route selection scheme which provides paths for multiple video streams with the least interference and an optimization algorithm that determines the compression rates depending on the network condition.

In urban vehicular wireless environments, although rebroadcasting by vehicles can possibly increase the number of vehicles receiving the traffic warning messages, redundancy, contention, and packet collisions due to simultaneous forwarding (usually known as the broadcast storm problem), can occur. The paper entitled "A Street Broadcast Reduction Scheme (SBR) to Mitigate the Broadcast Storm Problem in VANETs" presents street broadcast reduction (SBR), a novel scheme that mitigates the broadcast storm problem in VANETs. Simulation results show that SBR combined with the location-based scheme outperforms other schemes in high density urban scenarios, yielding a lower percentage of blind vehicles while drastically alleviating the broadcast storm problem.

The paper entitled "Performance Analysis of OFDM Repeater Networks with Spatial Fading Correlation" analyzes the performance of the OFDM diversity systems with the spatial fading correlation when the network includes an RF repeater. In this study, a multipath channel is constructed by the repeated signal instead of natural multipath and the system performance with spatial correlation per subcarrier is evaluated. Numerical results indicate that the time shifted sampling (TSS) technique which relies on multipath diversity is also effective in reducing the effect of correlation for the multipath channels constructed by the repeaters.

The paper "Enhancing Data Rates of TH-UWB Systems Using M-ary OPPM-BPSM Modulation Scheme: A System Perspective M-ary OPPM-BPSM" proposes and studies a combined modulation scheme for time hopping ultra wideband (THUWB) radio system based on orthogonal pulse position modulation (OPPM) and biorthogonal pulse shape modulation (BPSM). The proposed scheme offers a high data rate with satisfactory system performance by using high-level multi-dimensional modulation schemes. The performance is analyzed by using characteristic function and Gaussian approximation in additive white Gaussian and multipath scenarios, respectively. As demonstrated, the proposed scheme reduces multiple access interference considerably in multi-user environments and the theoretical analysis is validated by simulation results.

The paper "Cross-layer Fast Link Adaptation for MIMO-OFDM Based WLANs" considers the use of cross-layer fast link adaptation (FLA) for WLANs employing a MIMO-OFDM physical layer. A packet error rate (PER) based FLA technique that, without loss of generality, makes use of the exponential effective SNR mapping (EESM) is proposed. Results show that both PER- and BER-based FLA techniques optimize the data throughput while satisfying prescribed quality of service constraints. Channel estimation errors have also been considered, revealing the importance of good channel estimators in order for FLA strategies to work satisfactorily.

The paper entitled "Adaptive Self-learning Resource Allocation Scheme for Unlicensed Users in High-Rate UWB Systems" investigates a dynamic sub-band allocation for spectrum sharing in the multiuser OFDM-UWB systems while respecting the system constraints and the distributed MAC architecture. A dynamic spectrum allocation scheme is proposed and analyzed to derive a multiuser optimal allocation solution. Based on an adaptive and lowcomplexity approach, the proposed scheme shares the available resources among unlicensed UWB users while taking into consideration the QoS requirements and the channel conditions. 
This new approach improves the system performance compared to WiMedia solution and offers a considerable gain for users that have strict QoS requirements.

An efficient approach for organizing large ad hoc networks is to divide the nodes into multiple clusters and designate, for each cluster, a cluster head which is responsible for holding inter-cluster control information. On the one hand, the cluster head has a dominant position in front of the others because it manages the connectivity and has access to other node's sensitive information. But on the other hand, the cluster head role also has some associated costs. Hence, in order to prevent malicious nodes from taking control of the group in a fraudulent way and avoid selfish attacks from suitable nodes, the cluster head needs to be elected in a secure way. The paper entitled "A Fair and Secure Cluster Formation Process for Ad Hoc Networks" presents a novel solution that guarantees the cluster head is elected in a cheat-proof manner.

Acknowledgments We are thankful to all those authors who considered submitting their work to this Special Issue, irrespective of whether their papers could be accepted or not. We are thankful to all the Referees, who painstakingly reviewed the papers. Without their hard work and dedication, it would not have been possible to select these high quality papers within the time limits of this Special Issue. We are extremely grateful to the Editor-in-Chief of this Journal, Professor Ramjee Prasad, for supporting the launch of this Special Issue. Many thanks are also due to Mrs. Cindy Zitter of Springer for her help and support.

\section{Author Biographies}

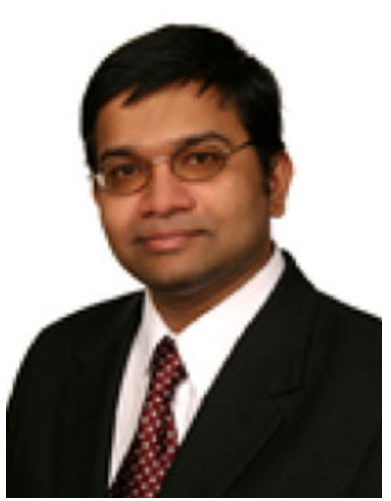

Sudip Misra is an Assistant Professor in the School of Information Technology at the Indian Institute of Technology Kharagpur, India. Prior to this he worked in Cornell University (USA) Yale University (USA), Nortel Networks (Canada) and the Government of Ontario (Canada). He received his Ph.D. degree in Computer Science from Carleton University, in Ottawa, Canada, and the masters and bachelors degrees respectively from the University of New Brunswick, Fredericton, Canada, and the Indian Institute of Technology, Kharagpur, India. Dr. Misra has several years of experience working in the academia, government, and the private sectors in research, teaching, consulting, project management, architecture, software design and product engineering roles. His current research interests include algorithm design and engineering for telecommunication networks, software engineering for telecommunication applications, and computational intelligence and soft computing applications in telecommunications. Dr. Misra is the author/editor of over 100 scholarly research papers and books. He has won five research paper awards in different conferences. He was also the recipient of several academic awards and fellowships such as the (Canadian) Governor General's Academic Gold Medal at Carleton University, the University Outstanding Graduate Student Award in the Doctoral level at Carleton University. In 2008, he was conferred The National Academy of Sciences, IndiaSwarna Jayanti Puraskar (Golden Jubilee Award). He was also awarded the Canadian Government's prestigious NSERC Post Doctoral Fellowship. His biography was also selected for inclusion in the 2006-2007 edition of Marquis Who's Who in Science and Engineering, and the 25th Edition of the Marquis Who's Who in the World, CA, USA. A mention about him and his work has also appeared in the November 4, 2006 issue of the Ottawa Citizen newspaper. Dr. Misra is the Editor-in-Chief of two journals-the International Journal of Communication Networks and Distributed Systems (IJCNDS) and the International Journal of Information and Coding Theory (IJICoT), UK. He is an Associate Editor of the Telecommunication Systems Journal (Springer SBM), Security and Communication Networks Journal (Wiley), International Journal of Communication Systems (Wiley), and the EURASIP Journal of Wireless Communications and Networking. He is also an Editor/Editorial Board Member/Editorial Review Board Member of the IET Communications Journal, Computers and Electrical Engineering Journal (Elsevier), International Journal of Internet Protocol Technology, the International Journal of Theoretical and Applied Computer Science, the International Journal of Ad Hoc and Ubiquitous Computing, Journal of Internet Technology, and the Applied Intelligence Journal (Springer). Dr. Misra is an editor of six books in the areas of wireless ad hoc networks, wireless sensor networks, wireless mesh networks, communication networks and distributed systems, network reliability 
and fault tolerance, and information and coding theory, published by reputed publishers such as Springer and World Scientific. He was invited to chair several international conference/workshop programs and sessions. He has been serving in the program committees of over a dozen international conferences. Dr. Misra was also invited to deliver keynote lectures in around a dozen international conferences in USA, Canada, Europe, Asia and Africa.

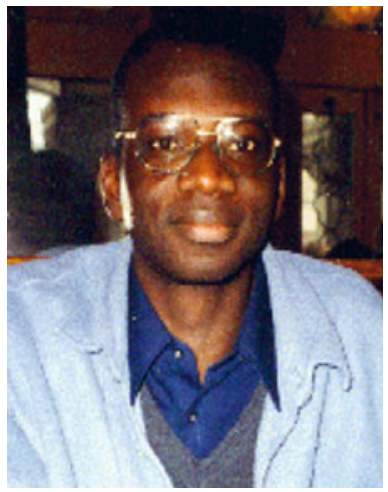

Issa Traore received an Aircraft Engineer Degree from Ecole de 1'Air in Salon de Provence (France) in 1990, and successively two Master Degrees in Aeronautics and Space Techniques in 1994, and in Automatics and Computer Engineering in 1995 from Ecole Nationale Superieure de l'Aeronautique et de l'Espace (E.N.S.A.E), Toulouse, France. In 1998, Traore received a Ph.D. in Software Engineering from Institute Nationale Polytechnique (INPT)-LAAS/CNRS, Toulouse, France. From June to October 1998, he held a post-doc position at LAASCNRS, Toulouse, France, and Research Associate (November 1998May 1999), and Senior Lecturer (June-October 1999) at the University of Oslo. Since November 1999, he has joined the Faculty of the Department of Electrical and Computer Engineering, University of Victoria, Canada. He is currently an Associate Professor. His research interests include distribution systems, behavioral biometrics systems, intrusion detection systems, software security metrics, and software quality engineering. He is the founder and coordinator of the Information Security and Object Technology (ISOT) Lab (http://www.isot.ece.uvic.ca).

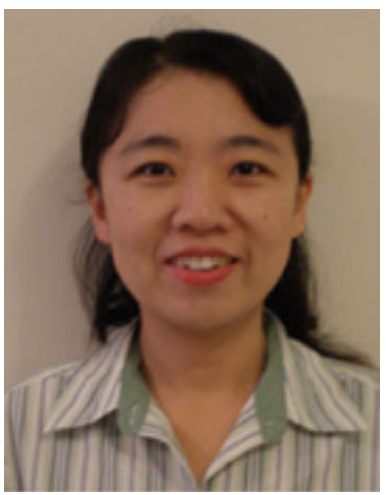

Wei Song received her Ph.D. degree in electrical and computer engineering from the University of Waterloo, Canada, in 2007. Since 2008, she has been supported by the Natural Science and Engineering Research Council (NSERC) of Canada and worked as a postdoctoral research fellow at the Department of Electrical Engineering and Computer Sciences, University of California, Berkeley. In July 2009, she joined the Faculty of Computer Science, University of New Brunswick, as an assistant professor. She received a Top 10\% Award from IEEE Workshop on Multimedia Signal Processing (MMSP) 2009, an NSERC postdoctoral fellowship in 2008, and a Best Paper Award from IEEE Wireless Communications and Networking Conference (WCNC) 2007. Her current research interests include the interworking of cellular networks and wireless local area networks (WLANs), resource allocation for heterogeneous wireless networks, vehicular ad hoc networks, and cross-layer optimization for multimedia quality-of-service (QoS) provisioning. Dr. Song has been actively participating in professional activities. She has served the Technical Program Committee for various international conferences including IEEE INFOCOM, IEEE ICC, IEEE GLOBECOM, and IEEE WCNC. Particularly, she was the system administrator for IEEE Transactions on Vehicular Technology and co-chaired the Wireless Access Track of IEEE Vehicular Technology Conference (VTC), Fall 2010. She is also an Editor of International Journal of Communication Networks and Distributed Systems and a co-guest editor of Springer Wireless Personal Communications Journal for the special issue on adaptive communications in wireless networks. 